[3] Maricq HR. Wide-field capillary microscopy[J]. Arthritis Rheum, 1981, 24(9): 1159-1165.

[4] Pavlov-Dolijanovic S, Damjanov NS, Stojanovic RM, et al.Scleroderma pattern of nailfold capillary changes as predictive value for the development of a connective tissue disease:a follow-up study of 3,029 patients with primary Raynaud's phenomenon[J]. Rheumatol Int, 2012,32(10):3039-3045.

[5] Marino Claverie L, Knobel E, Takashima L, et al.Organ involvement in Argentinian systemic sclerosis patients with "late" pattern as compared to patients with "early/active" pattern by nailfold capillaroscopy[J]. Clin Rheumatol, 2013, 32(6):839-843.

[6] Cutolo M, Grassi W, Matucci Cerinic M. Raynaud's phenomenon and the role of capillaroscopy[J]. Arthritis Rheum,2003, 48(11):3023-3030.

Disclosure of Interest: None declared

DOI: 10.1136/annrheumdis-2017-eular.3449

\section{AB0680 ANALYSIS THE CAUSES AND COUNTERMEASURES OF IGNORING SWALLOWING DYSFUNCTION IN PATIENTS WITH POLYMYOSITIS AND DERMATOMYOSITIS}

Y. Wen, Q. MinLi, L. Yan. Department of Rheumatology, Third Affiliated Hospital of Sun Yat-sen University, Guangzhou, China

Background: The patients with Polymyositis and Dermatomyositis always ignored swallowing dysfunction and most of them eventually had no enough knowledge of this. For this situation, to find out the reasons and take appropriate measures was necessary.

Objectives: To analyze the causes of ignoring swallowing dysfunction in patients with Polymyositis and Dermatomyositis and to explore the corresponding preventive measures.

Methods: The clinical data of 47 patients with Polymyositis and Dermatomyositis in hospital from September 2012 to December 2013 was analyzed retrospectively. The swallowing function was evaluated by the water swallow test, and the patients' knowledge of swallowing dysfunction was surveyed.

Results: Only 2 patients complained of choking during swallowing, with ignorance rate of $95.74 \%$. Positive rate was $40.43 \%$ in water swallow test, of which grade II dysphagia proposition was $58 \%$, III grade was $32 \%$, IV grade was $10 \%$. $100 \%$ of patients believed that sternal obstruction or dysphagia as swallowing dysfunction. $89.36 \%$ of patients didn't think drinking water with bucking as swallowing dysfunction.

Conclusions: The symptoms of limb weakness in patients with Polymyositis and Dermatomyositis may obscure the presence of dysphagia. In addition, the patients do not have enough knowledge about dysphagia that to neglect the swallowing dysfunction. To improve detection rate of swallowing dysfunction in patients with Polymyositis and Dermatomyositis, earching detailed history by listing dysphagia performance and providing water swallow test is necessary.

\section{References:}

[1] Comparison between swallowing-related and limb muscle involvement in dermatomyositispatients. Kim SJ, Han TR, Jeong SJ, Beom JW. Scand J Rheumatol. 2010 Aug;39(4):336-40.

Disclosure of Interest: None declared

DOI: 10.1136/annrheumdis-2017-eular.3125

\section{AB0681 HIDDEN GENERALIZED EDEMA IN INFLAMMATORY MYOPATHY; GENERALIZED EDEMA IS AN UNRECOGNIZED CLINICAL FEATURE OF MYOSITIS?}

Y. Namiki ${ }^{1}$, K. Kurasawa ${ }^{2}$, A. Tanaka ${ }^{2}$, R. Yamazaki ${ }^{1}$, H. Okada ${ }^{2}$, S. Arai ${ }^{2}$, T. Owada ${ }^{1}$, R. Maezawa ${ }^{1}$, M. Arima ${ }^{1} .{ }^{1}$ Department of Rheumatology, Dokkyo Medical University, Mibu, Tochigi; ${ }^{2}$ Department of Rheumatology, Dokkyo Medical University, Mibu, Tochigi, Japan

Background: Dermatomyositis (DM)/ polymyoitis (PM) are systemic diseases characterized by muscle inflammation, which shows varieties of clinical symptoms and signs. We have experienced cases of DM/PM with generalized edema as reported previously by others (1). Moreover, we found that there were many myositis patients who lost their body weight (BW) after starting of high dose glucocorticoid (GC) therapy. Thus, we hypothesized that hidden generalized edema is a characteristic clinical feature of myositis.

Objectives: To determine whether generalized edema is a hidden clinical feature of myositis. If so, what myositis patients have the feature.

Methods: The study was a retrospective observation study. The subjects were consecutive 67 of DM/PM and 53 of SLE patients who diseases for the first time, admitted our department from April 2007 to September 2016 and received immunosuppressive therapy including over $30 \mathrm{mg} /$ day GC. The patients were excluded who had cardiogenic or nephrogenic edema or whose BW data was not available. To detect hidden generalized edema caused by inflammation, we examined the change in BW within 2 weeks after starting immunosuppressive therapy. The clinical features of DM/PM patients with/without BW change were examined through reviewing medical record.

Results: The included subjects were DM/PM 66 patients (M/F; 18/48 with a mean age of $59.4 \mathrm{y}$.) and SLE 40 patients (M/F;14/26 with a mean age of $49.8 \mathrm{y}$.). The body weight of DM/PM and SLE were $56.4 \pm 14.0$ and $54.7 \pm 10.9 \mathrm{~kg}$, respectively. Decrease in BW within 2weeks after starting the therapy were $3.02 \pm 2.99 \mathrm{~kg}$ of
DM/PM and $0.85 \pm 2.87 \mathrm{~kg}$ of SLE, which was larger in DM/PM compared to SLE (Fig A). The numbers of patients who lost BW more than $2 \mathrm{~kg}$ within the 2 weeks were 42 in DM/PM (64\%) and 14 in SLE (35\%).

Serum albumin levels were slightly decreased by $0.18 \mathrm{~g} / \mathrm{dl}(0.06$ to $0.30 ; 95 \% \mathrm{Cl})$ in DM/PM, while no significant change was detected in SLE.

In myositis, change in BW was similar among DM, amyopathic DM (ADM) and PM (Fig.B). Moreover, no differences were found in the change of BW between patients with and without male sex, malignancy, interstitial pneumonia, anti-ARS $\mathrm{Ab}$ and anti-MDA5Ab. Additionally, between patients with and without BW loss more than $2 \mathrm{~kg}$, no differences were found in age, serum TP, Alb and CRP levels before and after treatment and prognosis.

A $\mathrm{Kg}$
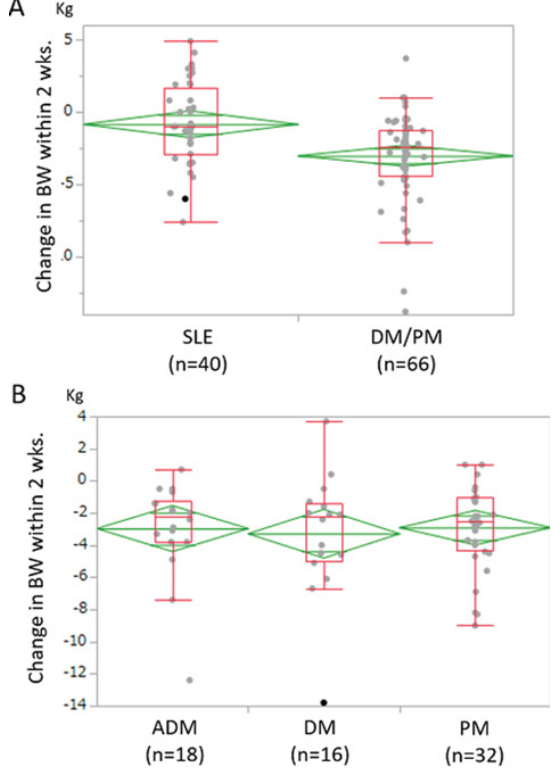

Conclusions: DM/PM patients lose BW by immunosuppressive therapy including GC, which indicates the existence of hidden generalized edema that might be a characteristic clinical feature in inflammatory myopathy.

References:

[1] Tu J1, McLean-Tooke A, Junckerstorff R. Increasing recognition of dermatomyositis with subcutaneous edema - is this a poorer prognostic marker? Dermatol Online J. 2014;20:21244.

Disclosure of Interest: None declared

DOI: 10.1136/annrheumdis-2017-eular.4822

\section{AB0682 THE INCIDENCE RATE OF INFLAMMATORY MYOPATHIES IN SLOVENIA}

Ž. Rotar ${ }^{1}$, A. Hočevar ${ }^{1}$, M. Krošel ${ }^{1}$, S. Praprotnik ${ }^{1}$, M. Tomšič ${ }^{1,2}$. ${ }^{1}$ Department of Rheumatology, University Medical Centre Ljubljana; ${ }^{2}$ Faculty of Medicine, University of Ljubljana, Ljubljana, Slovenia

Background: Annual incidence rates of inflammatory myopathies (IM) vary widely from 1.16-19.0 per $10^{6}$ of adults. 1 Our aim was to, for the first time, determine the incidence rate of IM in our population.

Objectives: To determine the incidence rate of IM in our population.

Methods: We retrospectively collected incident cases of IM from 1 January 2005 to 31 December 2016 at our department of rheumatology which is a part of an integrated secondary/tertiary university teaching hospital that is the only referral center for two well defined regions representing roughly a third of the national adult population. Tertiary cases are referred to our department from the entire country. We identified the cases by searching the electronic patient records (PRs) for ICD-10 codes M05, M33-35, M60, G73.7, G72.4. The paper and electronic PRs were scrutinized to assess clinical, laboratory and histopathological data. Descriptive statistics was used to describe our group of patients. The adult population size of the two regions served by our department was obtained from the national statistics institute database. The annual incidence rate for IM was then calculated.

Results: During the 12-year observation period we identified 117 new cases of IM from a well-defined adult white Caucasian population aged 18 or above. 38 cases were excluded from analyses since they were referred to our department from outside the two regions we serve on the secondary and tertiary level. Thus, we analyzed 79 cases of IM (63\% female; median (IQR) age 67 (55-75) years; $44 \%$ ever smokers). The median time to diagnosis was 5 (IQR 3-12) months. We diagnosed $29 \%$ patients with dermatomyositis, $25 \%$ with anti-synthetase syndrome, $18 \%$ with polymyositis, $9 \%$ with statin induced necrotizing autoimmune myopathy, $9 \%$ with concomitant myositis as a part of connective tissue disease, $6 \%$ with paraneoplastic myositis, and $4 \%$ with undifferentiated myositis. The IM cases were most often diagnosed in the summer months (32.9\%), followed 
by spring $(24.1 \%)$, fall $(22.8 \%)$, and winter $(20.3 \%)$. The clinical and laboratory characteristics at presentation are shown in Table 1. Muscle biopsy was performed in $93 \%$ of patients. Notably, patients with swallowing difficulties had more commonly had Raynaud phenomenon (RR 3.7 (95\% Cl 1.4-9.4), p=0.008).

Table 1. The clinical and laboratory characteristics at presentation

\begin{tabular}{l|l}
\hline & $\#(\%)$ \\
\hline Myositis & $72(91)$ \\
\hline Skin rash & $42(53)$ \\
\hline Weight loss & $35(44)$ \\
\hline Interstitial lung disease & $29(37)$ \\
Swallowing difficulties & $31(39)$ \\
Arthritis & $20(25)$ \\
Raynaud phenomenon & $14(18)$ \\
Fever & $9(11)$ \\
\hline median ESR & $27(16-45)$ \\
\hline MEDIAN CRP & $7(1-22)$ \\
\hline Hep-2 test: ANA (21:160) & $41(52)$ \\
\hline Hep-2 test: nonspecific cytoplasmic IF & $20(25)$ \\
\hline Anti-ENAS & $43(54)$ \\
\hline \multicolumn{1}{c}{ aJo-1 } & $17(22)$ \\
\hline aPL-12 & $3(4)$ \\
aMI-2 & $2(2.5)$ \\
aPM/SCl & $5(6)$ \\
aUU1-RNP & $1(1.3)$ \\
ACA & $1(1.3)$ \\
\hline aRo & $1(1.3)$ \\
\hline aSRP & $6(8)$ \\
\hline aSAE & $1(1.3)$ \\
\hline 3HHMGCoaR & $1(1.3)$ \\
\hline RTE/HSE & $3(4)$ \\
\hline Histopathologically proven IM & $19(24)$ \\
\hline Legend: ACA - anti-centromere antibodies, ANA- \\
anti-nuclear antibodies, anti-ENAs - autoantibodies \\
against extractable nuclear antigens, CRP - C-reactive \\
protein, EMG - electromyogram, ESR - erythrocyte \\
sedimentation rate, RTE/HSE - rabbit thymus \\
extract/human spleen extract used in the in-house \\
anti-ENA counter-immuno-diffusion assay, IF - \\
immunofluorescence, IM - inflammatory myopathy. \\
\hline
\end{tabular}

Conclusions: The averaged 12-year annual incidence of IM in the population under study was $9.4(95 \% \mathrm{Cl} 7.5-11.8)$ per $10^{6}$ adults.

References:

[1] Meyer A, et al. Rheumatology 2015

Disclosure of Interest: None declared

DOI: 10.1136/annrheumdis-2017-eular.2688

\section{Spondyloarthritis - treatment}

\section{AB0683 TROUGH INFLIXIMAB LEVELS AND ANTI-INFLIXIMAB ANTIBODIES IN SPONDYLOARTHRITIS PATIENTS ON TREATMENT WITH LOW DOSE INFLIXIMAB: A SINGLE CENTRE CROSS-SECTIONAL STUDY}

A. Patil ${ }^{1}$, S.K. Upadhyaya ${ }^{1}$, R. Dawar ${ }^{2}$, B. Vaishnav ${ }^{1}$, N. Dadhaniya ${ }^{1}$, S.J. Gupta ${ }^{1}$, R. Handa ${ }^{1} .{ }^{1}$ Rheumatology; ${ }^{2}$ Microbiology, Indraprastha Apollo Hospitals, Delhi, India

Background: Infliximab (IFX) is an anti-TNF, chimeric, monoclonal antibody approved for use in refractory spondyloarthritis $(\mathrm{SpA})$. Studies done in patients with Rheumatoid arthritis ${ }^{1}$ and Inflammatory bowel disease ${ }^{2}$ have demonstrated the clinical utility of the measurement of serum trough IFX and antibodies to IFX (ATI). In India, many centres including ours use IFX at lower doses of $3-5 \mathrm{mg} / \mathrm{kg}$ and on demand IFX treatment without the use of the loading dose IFX in SpA patients $^{3}$. Data on the utility of measuring trough IFX and ATI levels and their correlation with disease activity in such group of patients is lacking.

Objectives: To evaluate the co-relation between trough Infliximab levels and disease activity measures, viz ASDAS ESR and ASDAS CRP in SpA patients on low dose IFX therapy

To compare the mean ASDAS- ESR/CRP scores between ATI positive and ATI negative patients

Methods: Thirty-nine adult spondyloarthrtitis patients in the age group of 1870 years, meeting the ASAAS classification criteria for peripheral and/or axial spondyloarthritis were recruited into the study. The inclusion criteria required the patients to have had received three or more infusions of IFX at $3-5 \mathrm{mg} / \mathrm{kg} / \mathrm{dose}$ over the past 6 to 9 months. Blood samples were collected between two to three months after the previous IFX infusion for the measurement of the ATI and the trough IFX levels using the Matriks Biotek Shikari Q-ATI ELISA and Q-IFLIXI ELISA kits respectively. At the same time, disease activity of the patients was quantitated using ASDAS ESR and ASDAS CRP scores.
Correlation between the ASDAS scores and the trough IFX levels was analysed by Pearson's product moment correlation assay. The difference in mean trough IFX and ASDAS scores between the ATI positive and ATI negative patients were assessed using Welch two sample t-test.

Results: There was a moderately significant negative correlation between the trough IFX levels and the ASDAS-ESR $(r=-0.69, p<0.001)$, ASDAS-CRP scores $(r=-0.67, p<0.001)$ (Fig 1). ATI positive patients in comparison to ATI negative, had significantly higher ASDAS ESR and ASDAS CRP scores (Table 1).

Table 1. Table showing differences in ASDAS scores between ATI positive and negative patients

\begin{tabular}{lccc}
\hline & ATI +ve patients & ATI -ve patients & P value \\
\hline Mean ASDAS ESR & 3.13 & 1.56 & $<0.001$ \\
Mean ASDAS CRP & 3.06 & 1.45 & $<0.001$ \\
\hline
\end{tabular}

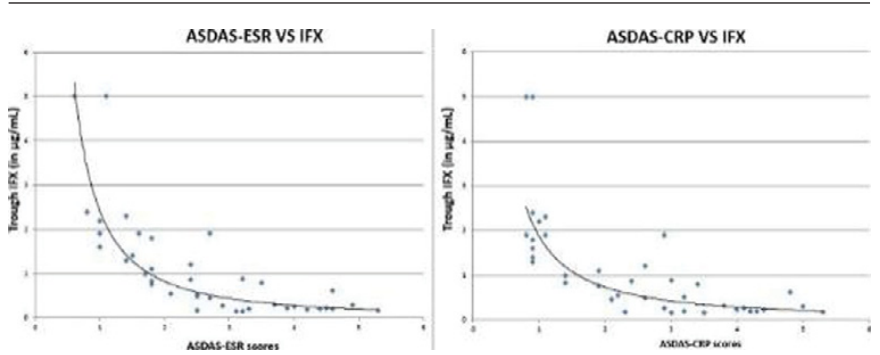

Conclusions: SpA patients from India on low dose, on demand IFX therapy, have both the trough IFX and ATI correlate significantly with the measures of disease activity. Therefore, these may be used in addition to clinical activity scores for a more cost effective on demand IFX therapy in SpA patients, especially in an expense constrained country like India.

References:

[1] Laine J, JokirantaT, Eklund K, Vakevainen M, Puolakka K. Cost effectiveness of routine measuring of serum drug concentrations and anti-drug antibodies in treatment of rheumatoid arthritis patients with TNF $\alpha$ blockers. Biologicals: Targets and therapy.2016;10:67-73.

[2] Pallagi Kunstar E, Farkas K, Szepes Z et al. Utility of serum TNF $\alpha$, infliximab trough level and antibody titres in inflammatory bowel disease. World $\mathrm{J}$ Gastroenterol. 2014;20:5031-5.

[3] Kumar A. Experience with anti-tumour necorsis factor- $\alpha$ therapy in India. APLAR journal of Rheumatology. 2006:9:136-41.

Disclosure of Interest: None declared

DOI: 10.1136/annrheumdis-2017-eular.2690

\section{AB0684 CLINICAL RESPONSE AND RADIOGRAPHIC PROGRESSION IN ANKYLOSING SPONDYLITIS PATIENTS UNDER ANTI-TNF THERAPY: IMPACT OF HIP INVOLVEMENT}

A.Y. Shimabuco ${ }^{1}$, F.M. Milanez ${ }^{1}$, J.C.B. Moraes ${ }^{1}$, G.V. Perico ${ }^{2}$,

C.R. Gonçalves $^{1}{ }^{1}$, M.G. Waisberg ${ }^{1}$, P.D. Sampaio-barros ${ }^{1}$, C.G.S. Saad ${ }^{1}$.

${ }^{1}$ Rheumatology Division, Faculdade de Medicina da Universidade de São Paulo, São Paulo; ${ }^{2}$ Unidade Radiológica Criciúma, Criciuma, Brazil

Background: Hip involvement is considered an important prognostic factor associated with radiographic progression in ankylosing spondylitis (AS) patients. However, there are no studies regarding hip involvement impact on clinical response and radiographic progression in AS patients under anti-TNF therapy.

Objectives: Compare clinical and radiographic progression in AS patients receiving anti-TNF therapy with and without moderate-severe hip involvement.

Methods: Forty-seven AS patients referred to receive anti-TNF treatment were included and classified according to baseline hip involvement based on Bath Ankylosing Spondylitis Radiology Hip Index (BASRI-Hip): none-minimal hip disease (hip grade $<3$ ) or moderate-severe disease (hip grade $\geq 3$ ). Demographic data, presence of HLA-B27, extra-articular involvement, DMARD and NSAID use, clinical and laboratorial disease parameters (BASDAI, BASMI, BASFI, ASQol, mSASSS and inflammatory markers) were assessed at baseline and two years after anti-TNF treatment.

Results: Thirty-four (72.3\%) patients were classified as none-minimal hip disease and $13(27.7 \%)$ as moderate-severe hip involvement. Both groups were similar at baseline considering age, HLA-B27, extra-articular involvement and comedication use. Laboratorial markers (ESR, CRP) and disease parameters (BASDAI, BASFI and mSASSS) showed no difference at baseline. Moderate-severe group had longer disease (10.0 \pm 7.6 vs. $14.9 \pm 8.6, \mathrm{P}=0.002$, years), higher BASMI $(3.8 \pm 2.4$ vs. $6.5 \pm 2.5, P=0.002)$ and lower $A S Q o L(13.7 \pm 4.4$ vs. $9.9 \pm 4.9, P=0.007)$. After two-years of anti-TNF therapy, both groups presented similar BASDAI response (delta BASDAI, $p=0.134$; final BASDAI, $p=0.324$ ) and an increase in mSASSS [no-minimal involvement: $13.6 \pm 18.3$ vs. $16.1 \pm 19.4, \mathrm{P}<0.001$ ); moderate-severe involvement: $21.7 \pm 19.9$ vs. $28.6 \pm 18.5, P=0.003$ ]. At final evaluation patients with moderate-severe hip involvement presented higher mSASSS (28.6 \pm 18.5 vs. $16.1 \pm 19.4, P=0.02$ ), despite similar delta BASDAI and final BASDAI.

Conclusions: Our study provides evidence that hip involvement did not impact on clinical response in AS patients under anti-TNF therapy but may have an effect on radiographic progression of these patients. 